\title{
Metodología de Sintonización de Parámetros del Estabilizador del Sistema de Potencia (PSS)
}

\author{
Pablo Verdugo Rivadeneira, Ing. \\ Jesús Játiva Ibarra, Ph.D. \\ Escuela Politécnica Nacional, Quito, Ecuador
}

\begin{abstract}
Resumen - Los sistemas eléctricos de potencia son susceptibles de perder su estabilidad debido a problemas asociados con la oscilación del rotor de los generadores sincrónicos. Para amortiguar estas oscilaciones se requiere la incorporación de un dispositivo en el sistema de excitación de estas máquinas. Este dispositivo es el estabilizador de sistemas de potencia (PSS).

En este trabajo se presenta un estudio del comportamiento de un sistema de prueba utilizando análisis modal. Con esta herramienta se puede llegar a conocer los modos de oscilación y su amortiguamiento así como la ubicación preliminar de un PSS en el sistema. Esta información es respaldada mediante un análisis de frecuencia en el modelo Generador - Barra Infinita efectuado en Simulink. Utilizando este modelo se logran obtener los valores de las constantes de tiempo del PSS que proporcionan una compensación de fase adecuada. Por otro lado, la ganancia del estabilizador se obtiene utilizando el método del lugar geométrico de las raíces.

Para comprobar la efectividad de la sintonización de los parámetros del PSS, se realizan simulaciones en el dominio del tiempo con el software DIgSILENT Power Factory.
\end{abstract}

Índices: Estabilizador de Sistemas de Potencia, Sintonización, Dominio del Tiempo y Frecuencia, Simulink, Power Factory

\begin{abstract}
Power electric systems are prone to lose its stability due to problems associated to synchronous generator rotor oscillations. In order to mitigate these oscillations a device into the machine excitation systems are required to incorporate. This device is the Power System Stabilizer PSS.

In this work a behavioral study of a test system utilizing modal analysis is presented. With this tool oscillation modes and its damping as well as a preliminary location of the PSS in the system is achieved. This information is supported by means of a frequency analysis in a bus infinite - generator system model made in Simulink. Utilizing this model PSS time constant values are obtained that gives adequate phase compensation. On the other hand, the PSS gain is attained by using the root locus technique.

In order to test the PSS tuning parameter effectiveness, time domain simulations with DIgSILENT Power Factory are performed.
\end{abstract}

Indexes: Power System Stabilizer, Tuning, Time and frequency domains, Simulink, Power Factory.

\section{INTRODUCCIÓN ${ }^{[1]}$}

La estabilidad de un sistema eléctrico de potencia se puede definir como la propiedad que le permite mantenerse en un estado de equilibrio bajo condiciones normales de operación y de regresar a un estado aceptable de equilibrio después de sufrir una perturbación.

Por su parte, la inestabilidad en un sistema de potencia puede manifestarse de diversas formas, dependiendo de su configuración y modo de operación. Una condición necesaria para la operación del sistema es que todos los generadores en línea permanezcan en sincronismo.

La habilidad que poseen las máquinas de un sistema para permanecer en sincronismo, corresponde a la estabilidad del ángulo del rotor. Esta estabilidad puede romperse ya sea por un desvío aperiódico del ángulo debido a la falta de torque sincronizante o a problemas de inestabilidad oscilatoria que tienen su origen en la falta de torque de amortiguamiento.

Los estabilizadores de sistemas de potencia son los dispositivos responsables de proporcionar una componente de torque de amortiguamiento a las máquinas con el fin de reducir las oscilaciones en el sistema causadas por pequeñas perturbaciones.

El comportamiento de un sistema dinámico se puede conocer mediante el análisis del espacio de estado. Las variables de estado pueden ser cantidades físicas en un sistema, como ángulo, velocidad, voltaje, o variables matemáticas asociadas con las ecuaciones diferenciales que describen la dinámica del sistema.

Con la solución de la ecuación característica de la matriz de estado, se obtienen los valores propios del sistema, los que contienen información valiosa de la estabilidad del mismo.

Un valor propio viene dado por:

$$
\lambda=\sigma \pm j \omega
$$

La parte real del valor propio $\sigma$ proporciona información acerca del amortiguamiento, mientras que la parte imaginaria $\omega$ provee la frecuencia de un modo de oscilación.

Cada valor propio tiene asociado un vector propio derecho y un vector propio izquierdo. Haciendo uso de estos vectores propios de manera simultánea se obtiene la 
matriz de participación, que proporciona una medida de la asociación entre las variables de estado y sus modos.

Los modos de oscilación se diferencian de acuerdo a rangos de frecuencia. En este trabajo, se consideran los modos locales y los modos interárea.

\section{A. Modos Locales}

Los modos locales están asociados con la oscilación de unidades en una planta de generación con respecto al resto del sistema de potencia en el rango de $1,0 \mathrm{~Hz}$ a 2,0 $\mathrm{Hz}$. Están localizados en una planta o una parte pequeña del sistema.

\section{B. Modos Interárea}

Los modos interárea están asociados con la oscilación de muchas máquinas en una parte del sistema contra máquinas ubicadas en otros lugares. Son causados por dos o más grupos de generadores que están interconectados por lazos débiles y oscilan a frecuencias de $1 \mathrm{~Hz}$ o menores.

\section{MODELO GENERADOR - BARRA INFINITA}

Se considera la representación linealizada para pequeña señal de un sistema con una máquina, como se muestra en la figura 1. En forma general, este modelo representa el comportamiento dinámico de un sistema, que incluye las relaciones electromecánicas entre el torque de aceleración y las desviaciones de velocidad y ángulo, el amortiguamiento de la turbina, el torque sincronizante y la dinámica de flujo del generador. ${ }^{[2]}$

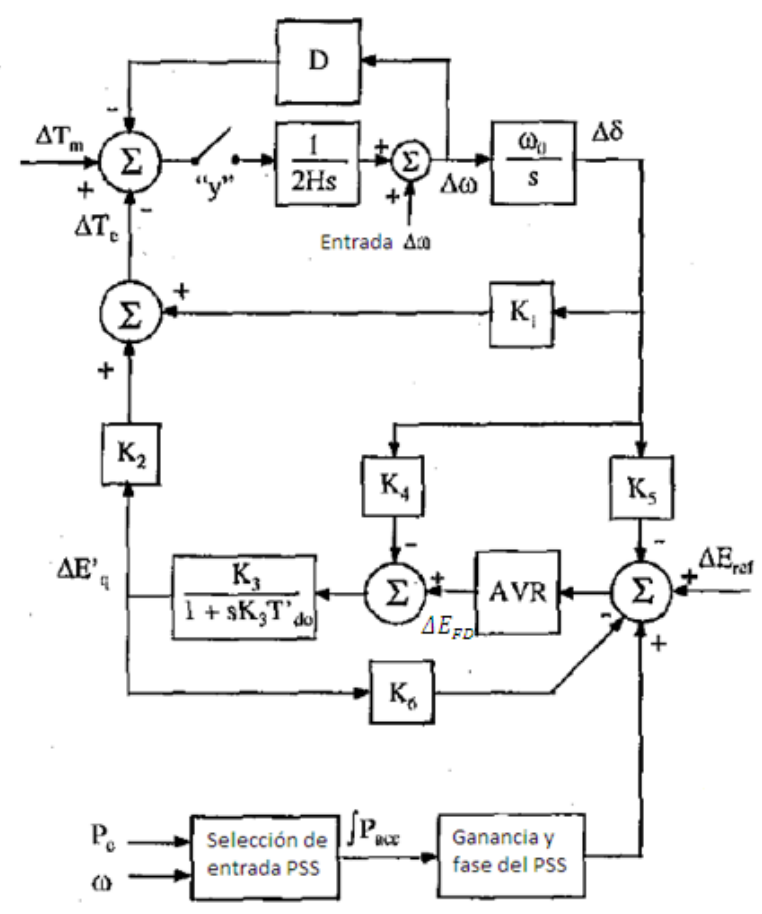

Fig. 1 Modelo Generador - Barra Infinita considerando AVR y PSS de doble entrada
De este modelo, se obtiene la respuesta de frecuencia del sistema alrededor de un punto de operación. Específicamente, se requiere conocer la función de transferencia desde la entrada a la excitatriz hasta la señal del torque eléctrico. Esta función de transferencia proporciona la información acerca del retraso de fase que existe en el sistema y por ende la compensación que debe ser provista por el PSS.

Para obtener las constantes " $\mathrm{K}$ " se requiere básicamente; los parámetros del generador, los resultados de corridas de flujos de potencia y cortocircuitos, con los que se obtiene el equivalente Thevenin del sistema para representar un generador conectado a una barra infinita a través de una línea de transmisión.

El modelo simplificado se desarrolla bajo las siguientes consideraciones: El efecto de los devanados de amortiguamiento es insignificante y el efecto de saturación del hierro no es importante. Con estas premisas se procede a simplificar las ecuaciones. Se presenta un ejemplo de cálculo para la constante $\mathrm{K} 1$ :

$$
\Delta T_{E}=K_{1} \Delta \delta+K_{2} \Delta \psi_{f d}
$$

$K_{1}=\Delta T_{E} / \Delta \delta$ para $\psi_{f d}$ constante

$K_{2}=\Delta T_{E} / \Delta \psi_{f d}$ para un ángulo de rotor $\delta$ constante

$$
\begin{aligned}
& K_{1}=\frac{E_{B} E_{q 0}}{D}\left(R_{T} \sin \delta_{0}+X_{T d} \cos \delta_{0}\right)+\frac{E_{B} i_{q 0}}{D}\left(X_{q}-\right. \\
& \left.X_{d}\right)\left(X_{T q} \sin \delta_{0}+R_{T} \cos \delta_{0}\right)
\end{aligned}
$$

Despreciando la resistencia de la línea de transmisión, se tiene:

$$
\begin{aligned}
& K_{1}=\frac{E_{B} E_{q 0}}{D}\left(X_{T d} \cos \delta_{0}\right)+\frac{E_{B} i_{q 0}}{D}\left(X_{q}-X_{d}^{\prime}\right)\left(X_{T q} \sin \delta_{0}\right) \\
& D=X_{T q} X_{T d} \\
& K_{1}=\frac{E_{B} E_{q 0} \cos \delta_{0}}{X_{T q}}+\frac{E_{B} i_{q 0}\left(X_{q}-X_{d}\right) \sin \delta_{0}}{X_{T d}}
\end{aligned}
$$

Para las demás expresiones se aplica un procedimiento similar y se remplazan ciertos términos a fin de que las variables de las ecuaciones puedan obtenerse fácilmente mediante una simulación del sistema de potencia.

El parámetro $\mathrm{X}_{\mathrm{E}}$ se refiere a la reactancia de la línea de transmisión que conecta al generador con la barra infinita. Este valor se obtiene mediante el equivalente Thevenin del sistema realizando un análisis de cortocircuitos en la barra del generador considerado.

$$
\begin{aligned}
K_{2} & =\frac{X_{T q} i_{q 0} \sin \delta_{0}}{X_{T d}}=\frac{E_{B} \sin \delta_{0}}{X_{T d}} \\
K_{3} & =\frac{X_{T d}}{X_{E}+X_{d s}} \\
K_{4} & =\frac{\left(X_{d s}-X_{d}\right)}{X_{T d}} E_{B} \sin \delta_{0} \\
K_{5} & =\frac{X_{q} E_{t d 0}}{X_{T q} E_{t}} E_{B} \cos \delta_{0}+\frac{X_{d} E_{t q 0}}{X_{T d} E_{t}} E_{B} \sin \delta_{0} \\
K_{6} & =\frac{X_{E} E_{q 0}}{X_{T d} E_{t}}
\end{aligned}
$$




\section{METODOLOGÍA DE SINTONIZACIÓN DEL PSS}

Se describe la metodología para sintonizar los parámetros del PSS que utiliza como entrada la integral de la potencia de aceleración, como se indica en la figura 2. Esta señal se obtiene mediante el tratamiento de las señales de velocidad y potencia eléctrica.

El primer paso para sintonizar los parámetros de un PSS es conocer el comportamiento de un sistema sin la presencia del estabilizador. Para esto se realiza un análisis modal utilizando el programa Power Factory de DIgSILENT, que permite conocer los valores propios de un sistema y los factores de participación de los generadores en determinado modo.

Los valores propios permiten conocer los modos de oscilación presentes en el sistema y si estos modos se encuentran bien o mal amortiguados. En otras palabras, permite conocer si se requiere o no la implementación de un PSS en el sistema.
Por otro lado, a través de los factores de participación se puede conocer la forma en la que se encuentran oscilando los generadores de un sistema. Adicionalmente, a través de esta información se llega a tener un indicio acerca de la ubicación de un PSS. Esta información se confirma a través de la respuesta de frecuencia de los generadores candidatos, incorporados al modelo Generador - Barra Infinita. Un buen enfoque, involucra la instalación del PSS en el generador en el que se obtiene la mayor descompensación de fase.

La figura 3 muestra el sistema de prueba empleado para realizar la sintonización del PSS. Una vez seleccionado el generador en el que se conoce que la instalación del PSS tendrá el mayor efecto, se procede a la sintonización de parámetros.

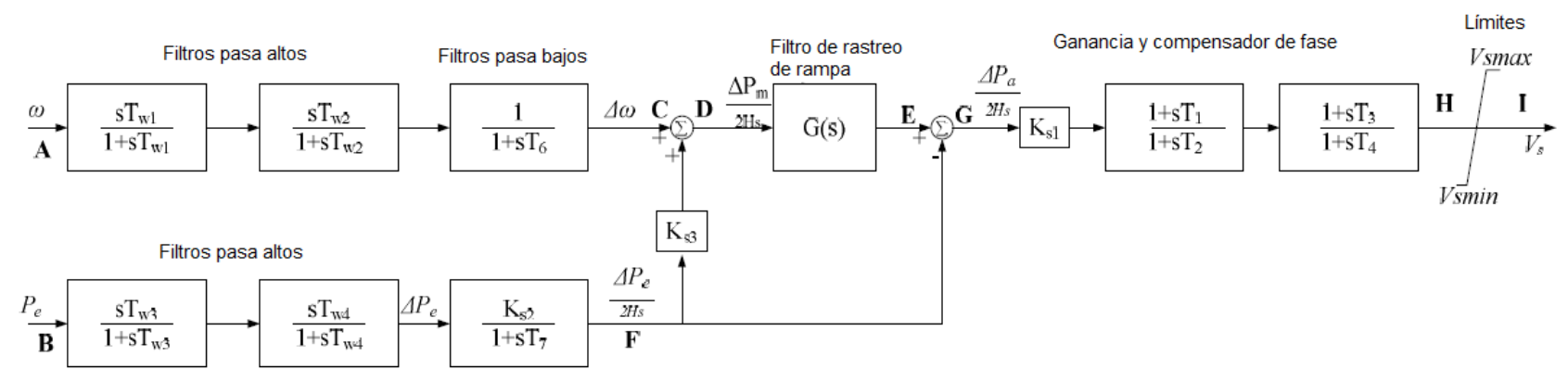

Fig. 2 Diagrama de bloques del estabilizador del sistema de potencia PSS2A

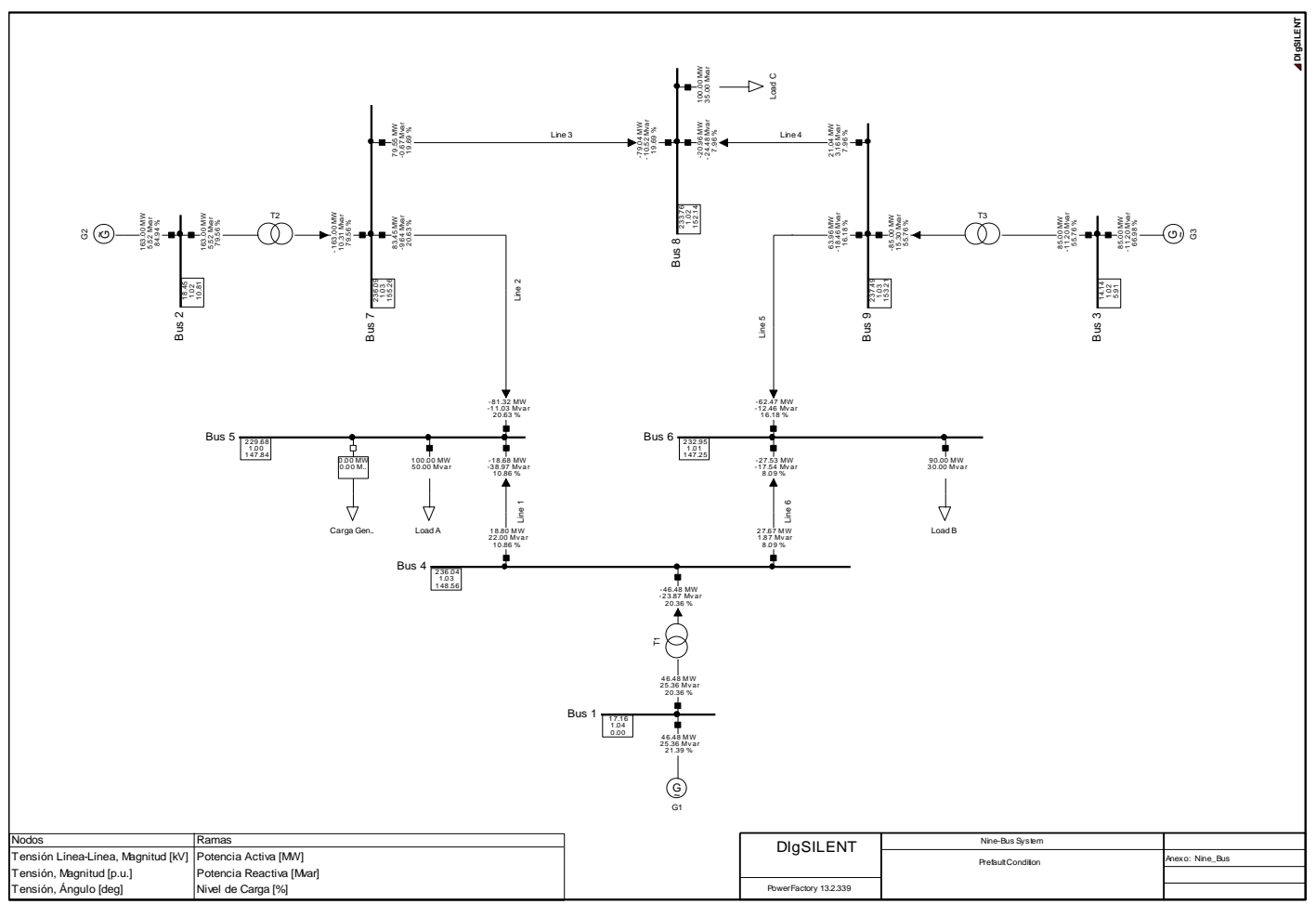

Fig. 3 Sistema de prueba de nueve barras 


\section{A. Sintonización de las Etapas de Compensación}

La respuesta de frecuencia del sistema en ausencia del PSS, indica cual es la compensación de fase que debe ser provista por este dispositivo. La respuesta típica de frecuencia de los bloques de compensación de un PSS se muestra en la figura 4.

La respuesta de frecuencia del PSS debe proveer una compensación de fase adecuada al sistema. Esto se logra a través del ajuste de las constantes de tiempo $T_{1}, T_{2}, T_{3}$, $y T_{4}$ del compensador de fase tomando en cuenta los conceptos de frecuencia centro y razón entre dichas constantes, ecuaciones (11) y (12). Una mala sintonización puede ocasionar una sobrecompensación en el sistema que se deriva en un problema de inestabilidad.

$$
\begin{gathered}
f c=\frac{1}{2 \pi} \frac{1}{\sqrt{T_{1} T_{2}}} \\
n=\frac{T_{1}}{T_{2}}=\frac{T_{3}}{T_{4}}
\end{gathered}
$$

La respuesta de frecuencia del sistema compensado debe presentar un ligero retraso de fase. Con esto, se logra que el PSS además de aportar con la componente de torque de amortiguamiento, introduzca al sistema una componente adicional de torque sincronizante.

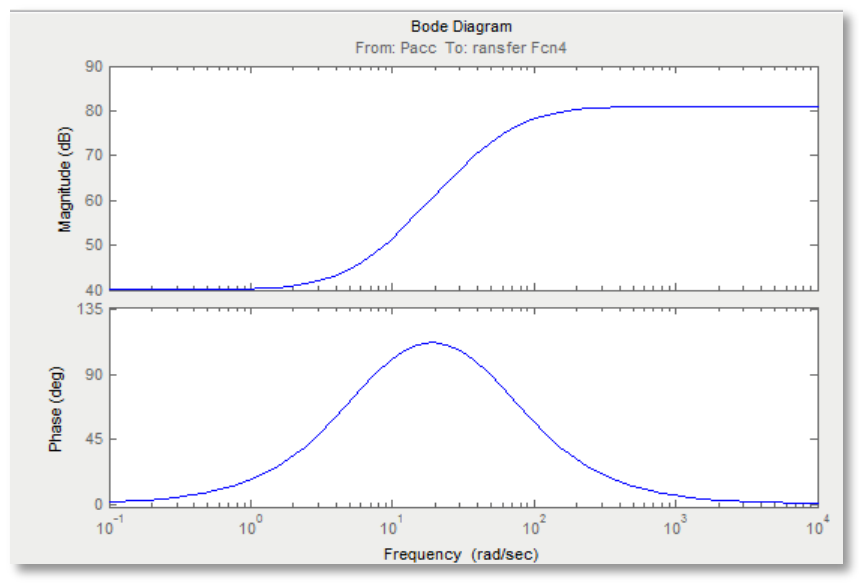

Fig. 4 Respuesta de frecuencia de los bloques de compensación de fase de un PSS

\section{B. Sintonización de la Ganancia}

Para obtener la ganancia del estabilizador se utiliza el método del lugar geométrico de las raíces.

Incrementando paulatinamente el valor de la ganancia, hasta evidenciar una condición de inestabilidad en el sistema en la cual se observe la migración de los valores propios hacia la parte positiva del plano $s$, se obtiene lo que se conoce como la ganancia de inestabilidad. En un PSS con entrada de potencia, la ganancia que proporciona el máximo amortiguamiento es la octava parte del valor de esta ganancia de inestabilidad. ${ }^{[3]}$

\section{SINTONIZACIÓN DE LOS PARÁMETROS DEL PSS}

En este apartado se expone la sintonización de los parámetros de un PSS2A para el sistema de prueba de tres generadores, aplicando la metodología descrita en III.

\section{A. Análisis del Sistema sin PSS}

A través del análisis modal efectuado en el sistema de prueba se obtienen los resultados de las figuras 5 y 6 , correspondientes a los valores propios y los factores de participación respectivamente.

A través de esta información se observa que el valor propio 16 y su conjugado, el valor propio 17, tienen una frecuencia de oscilación de $2,059 \mathrm{~Hz}$ y una razón de amortiguamiento de $4,93 \%$, lo que podría considerarse como un amortiguamiento pobre.

Adicionalmente, se puede observar, en base a la participación de los generadores, que el generador 1 se encuentra oscilando contra los generadores 2 y 3 . Dado que los generadores 1 y 2 tienen la mayor participación en estos modos, deben ser considerados como candidatos para la

\begin{tabular}{|c|c|c|c|c|c|c|c|c|c|c|c|}
\hline \multicolumn{3}{|c|}{ No. Valor Real/Imaginario } & \multicolumn{3}{|c|}{ No. Valor Real/Imaginario } & \multicolumn{3}{|c|}{ No. Valor Real/Imaginario } & \multicolumn{3}{|c|}{ No. Valor Real/Imaginario } \\
\hline $\begin{array}{l}1 \\
2 \\
3 \\
4 \\
5 \\
6 \\
7 \\
8\end{array}$ & $\begin{array}{r}0.000 \\
-257.517 \\
-254.541 \\
-252.209 \\
-50.808 \\
-16.784 \\
-16.784 \\
-19.264\end{array}$ & $\begin{array}{r}0.000 \\
0.000 \\
0.000 \\
0.000 \\
0.000 \\
33.413 \\
-33.413 \\
22.913\end{array}$ & $\begin{array}{r}9 \\
10 \\
11 \\
12 \\
13 \\
14 \\
15 \\
16\end{array}$ & $\begin{array}{r}-19.264 \\
-32.597 \\
-0.807 \\
-0.807 \\
-7.386 \\
-7.386 \\
-24.910 \\
-0.639\end{array}$ & $\begin{array}{r}-22.913 \\
0.000 \\
19.786 \\
-19.786 \\
22.642 \\
-22.642 \\
0.000 \\
12.939\end{array}$ & $\begin{array}{l}17 \\
18 \\
19 \\
20 \\
21 \\
22 \\
23 \\
24\end{array}$ & $\begin{array}{r}-0.639 \\
-0.000 \\
-2.348 \\
-3.103 \\
-9.647 \\
-15.946 \\
-14.926 \\
-10.000\end{array}$ & $\begin{array}{r}-12.939 \\
0.000 \\
0.000 \\
0.000 \\
0.000 \\
0.000 \\
0.000 \\
0.000\end{array}$ & $\begin{array}{l}25 \\
26 \\
27 \\
28 \\
29\end{array}$ & $\begin{array}{r}-0.833 \\
-10.000 \\
-0.833 \\
-10.000 \\
-0.833\end{array}$ & $\begin{array}{l}0.000 \\
0.000 \\
0.000 \\
0.000 \\
0.000\end{array}$ \\
\hline
\end{tabular}
instalación de un PSS.

Fig. 5 Valores propios del sistema de prueba

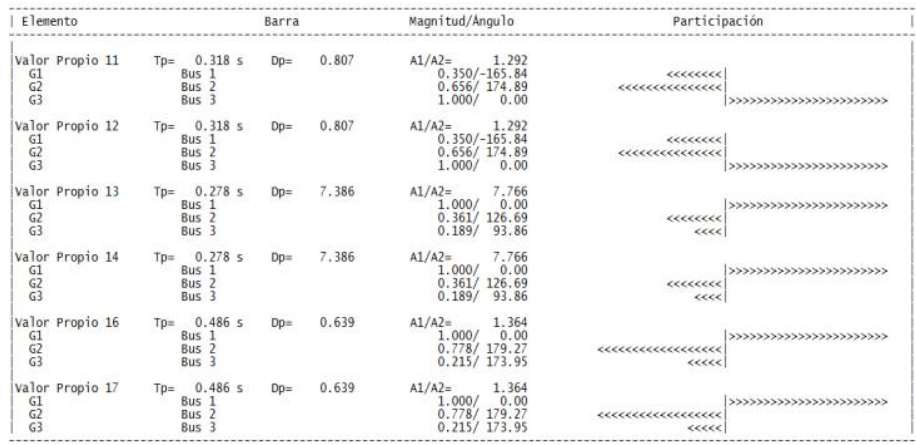

Fig. 6 Participación de los generadores en el sistema de prueba

Del análisis de respuesta de frecuencia, realizado en el modelo de la figura 7, se obtiene que el generador 2 es la mejor alternativa para colocar un PSS, ya que se evidencia claramente una disminución de las oscilaciones en el sistema, tras observar las simulaciones en distintas variables del sistema, como potencia eléctrica, frecuencia, ángulo del rotor, voltajes en las barras, flujos de potencia a través de las líneas.

El diagrama de Bode se obtiene a través de un proceso de linealización en el modelo implementado en Simulink. Se 
puede llegar a obtener la misma información, conociendo la función de transferencia del sistema. Sin embargo, este proceso resulta mucho más complicado.

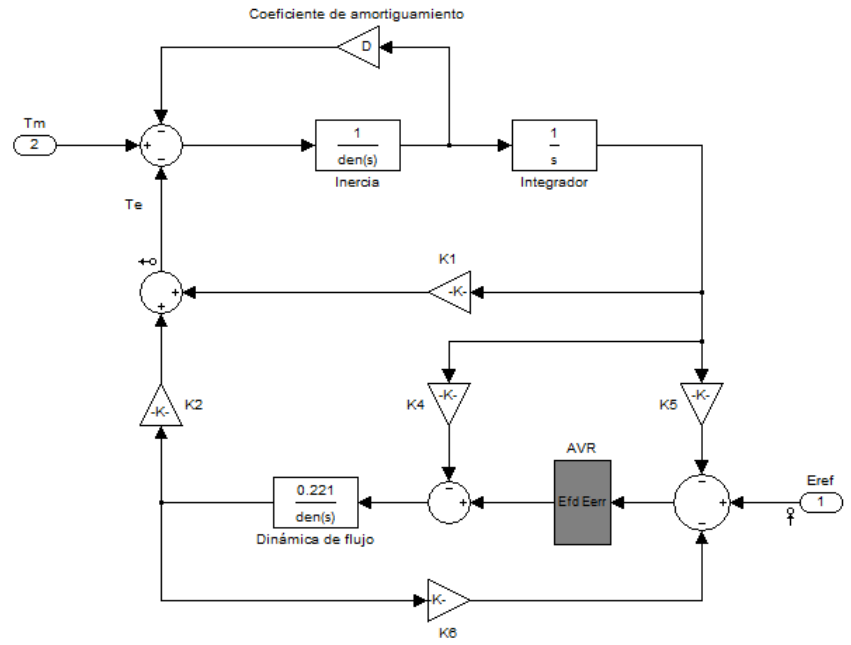

Fig. 7 Modelo implementado en Simulink

Para obtener los valores de las constantes " $K$ " se utiliza una hoja de cálculo, donde se consideran los parámetros del generador, potencia suministrada, voltaje en las barras, etc. Los resultados obtenidos de estas constantes se muestran en la Tabla 1.

Tabla 1 Constantes del Modelo Generador - Barra Infinita

\begin{tabular}{|c|r|}
\multicolumn{1}{c}{ Parámetro } & \multicolumn{1}{c}{ Valor } \\
\hline K1 & 1,84406213 \\
\hline K2 & 0,9054827 \\
\hline K3 & 0,2214364 \\
\hline K4 & 1,34916922 \\
\hline K5 & $-0,0499917$ \\
\hline K6 & 0,44910418 \\
\hline
\end{tabular}

Al ingresar estas constantes en el modelo implementado en Simulink, se obtiene la respuesta de frecuencia de la figura 8.

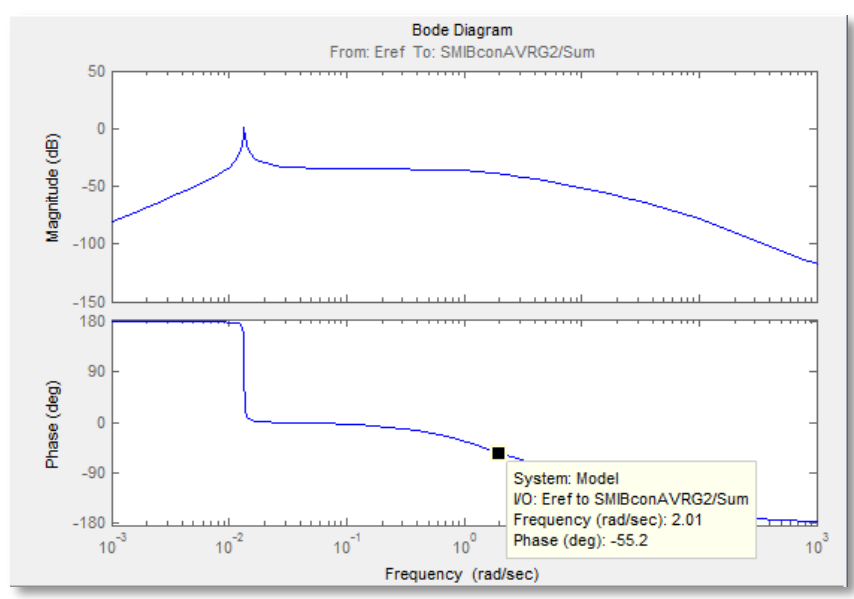

Fig. 8 Respuesta de frecuencia del sistema de prueba sin PSS
Como se puede observar, existe un retraso de fase de aproximadamente $55^{\circ}$ alrededor de los $2 \mathrm{~Hz}$. Con la compensación de fase provista por el PSS se obtiene la respuesta de la figura 9 .

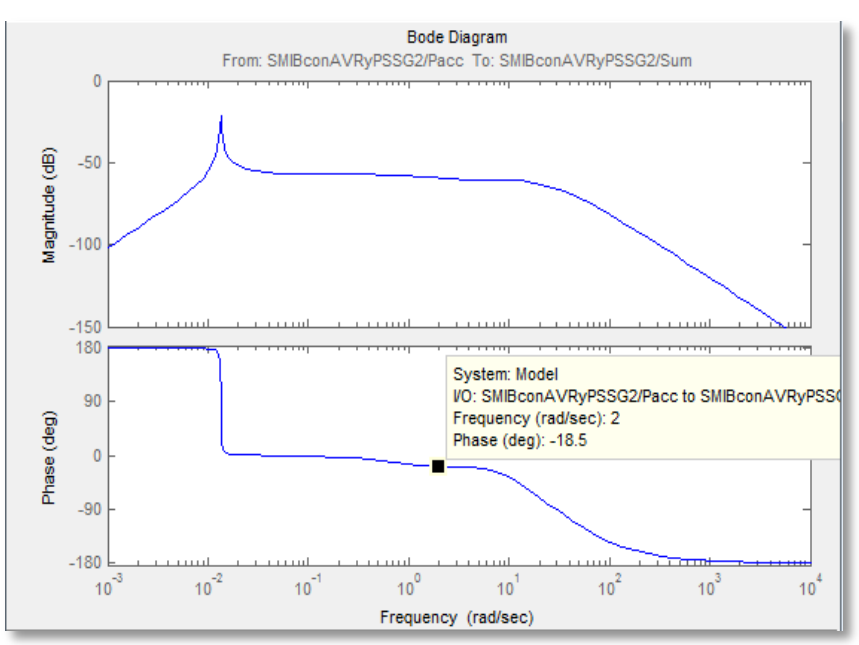

Fig. 9 Respuesta de frecuencia del sistema compensado

Las constantes de tiempo de los bloques de compensación de fase con los que se logra esa respuesta son: $\mathrm{T} 1=\mathrm{T} 3=0,27$ y $\mathrm{T} 2=\mathrm{T} 4=0,09$. Cabe mencionar que los parámetros de los filtros pueden encontrarse dentro del rango entre 1 y $20 \mathrm{~s}$.

Una vez establecida la compensación de fase adecuada, se procede con la sintonización de la ganancia. Al incrementar este valor paulatinamente, se observa que la migración de valores propios hacia la parte positiva del plano real se consigue con una ganancia de 1,6. Entonces, la ganancia que proporciona el máximo amortiguamiento en el PSS es de 0,2 .

Esta condición de inestabilidad, se observa en la figura 10. Vale indicar, que la ganancia de inestabilidad es el mínimo valor con el cual se observa valores propios con partes reales positivas.

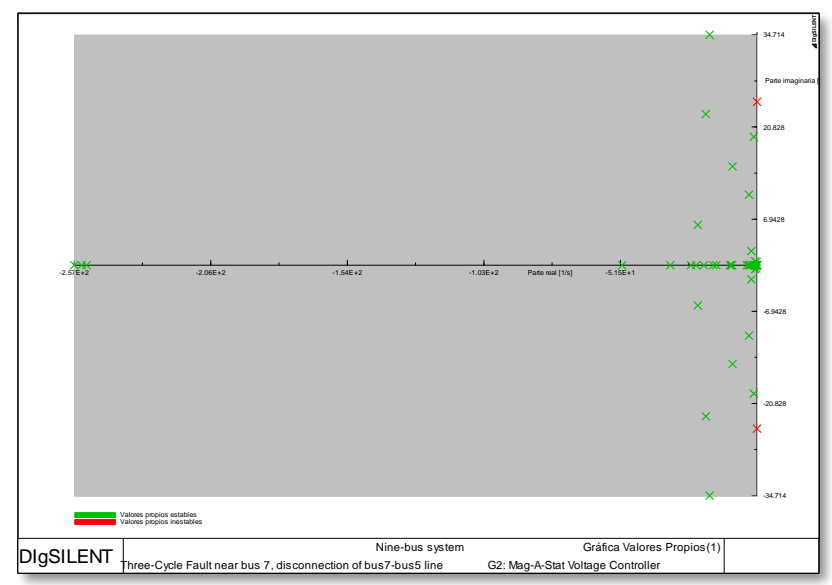

Fig. 10 Valores propios para la ganancia de inestabilidad Ks1=1,6 
Para comprobar la efectividad de la metodología de sintonización de los parámetros del estabilizador, se presentan los valores propios del sistema con el PSS en la figura 11.

\begin{tabular}{|rrr|rrr|rrr|rrr|}
\hline No. Valor Real/Inaginario | No. Valor Real/Imaginario & No. Valor Real/Imaginario & No. Valor Real/Imaginario \\
\hline 1 & 0.000 & 0.000 & 12 & -7.320 & -22.560 & 23 & -15.381 & 0.000 & 34 & -0.667 & -0.000 \\
2 & -257.664 & 0.000 & 13 & -2.003 & 19.886 & 24 & -9.638 & 0.000 & 35 & -10.000 & 0.000 \\
3 & -2544.858 & 0.000 & 14 & -2.003 & -19.886 & 25 & -5.216 & 2.387 & 36 & -0.833 & 0.000 \\
4 & -252.209 & 0.000 & 15 & -24.909 & 0.000 & 26 & -5.216 & -2.387 & 37 & -10.000 & 0.000 \\
5 & -50.808 & 0.000 & 16 & -21.126 & 5.299 & 27 & -5.609 & 0.000 & 38 & -0.833 & 0.000 \\
6 & -15.582 & 33.651 & 17 & -21.126 & -5.299 & 28 & -3.100 & 0.000 & 39 & 0.000 & 0.000 \\
7 & -15.582 & -33.651 & 18 & -1.912 & 11.217 & 29 & -2.348 & 0.000 & 40 & -20.000 & 0.000 \\
8 & -17.364 & 24.458 & 19 & -1.912 & -11.217 & 30 & -0.000 & 0.000 & 41 & 0.000 & 0.000 \\
9 & -17.364 & -24.458 & 20 & -6.804 & 11.199 & 31 & -0.665 & 0.056 & 42 & -10.000 & 0.000 \\
10 & -33.144 & 0.000 & 21 & -6.804 & -11.199 & 32 & -0.665 & -0.056 & 43 & -0.833 & 0.000 \\
11 & -7.320 & 22.560 & 22 & -16.216 & 0.000 & 33 & -0.667 & 0.000 & & & \\
\hline
\end{tabular}

Fig. 11 Valores propios del sistema con PSS

En base a los nuevos valores propios del sistema, se puede observar que todos los modos se encuentran bien amortiguados.

Adicionalmente, se realizan simulaciones en el dominio del tiempo considerando dos escenarios. Luego de cada simulación se incluye una tabla en la que se muestran los índices de desempeño, específicamente, el tiempo de establecimiento y el sobreimpulso de las señales, en los escenarios donde la obtención de estos parámetros resultó posible.

\section{B. Incremento de carga}

Este escenario plantea una pequeña perturbación en el sistema, asociada con el incremento de carga en una de sus barras. Se puede observar en los resultados mostrados en las figuras 12 y 13, que la instalación del PSS logra amortiguar las oscilaciones presentadas de manera efectiva. Los respectivos índices de desempeño se detallan en las tablas 2 y 3.

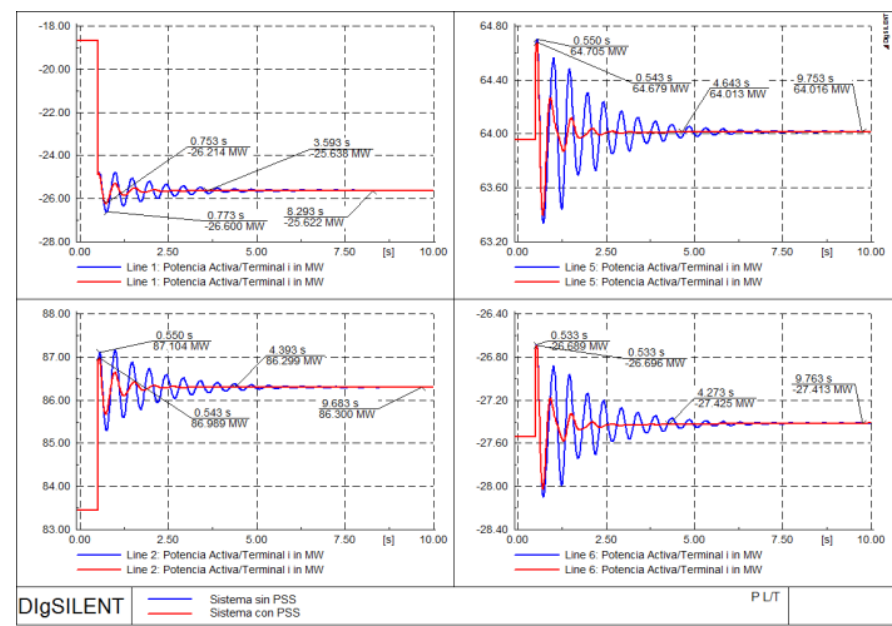

Fig. 12 Potencia eléctrica en las líneas de transmisión con incremento de carga

Se puede observar que al incorporar un PSS se produce una reducción considerable en el tiempo de establecimiento de las señales, así como también en el sobreimpulso de las mismas.
Tabla 2 Índices de desempeño de las señales de la Fig. 12

\begin{tabular}{|c|c|c|c|c|c|c|}
\hline \multicolumn{7}{|c|}{ Potencia activa en las líneas de transmisión } \\
\hline & \multicolumn{2}{|c|}{ Tiempo de Establecimiento [s] } & \multirow[t]{2}{*}{ Reducción [\%] } & \multicolumn{2}{|c|}{ Sobreimpulso [MW] } & \multirow[t]{2}{*}{ Reducción [\%] } \\
\hline & Sin PSS & Con PSS & & Sin PSS & Con PSS & \\
\hline Linea 1 & 8,293 & 3,593 & 56,674 & $-0,978$ & $-0,592$ & 39,468 \\
\hline Línea 2 & 9,683 & 4,393 & 54,632 & 0,804 & 0,689 & 14,303 \\
\hline Línea 5 & 9,753 & 4,643 & 52,394 & 0,689 & 0,663 & 3,774 \\
\hline Línea 6 & 9,763 & 4,273 & 56,233 & 0,724 & 0,717 & 0,967 \\
\hline
\end{tabular}

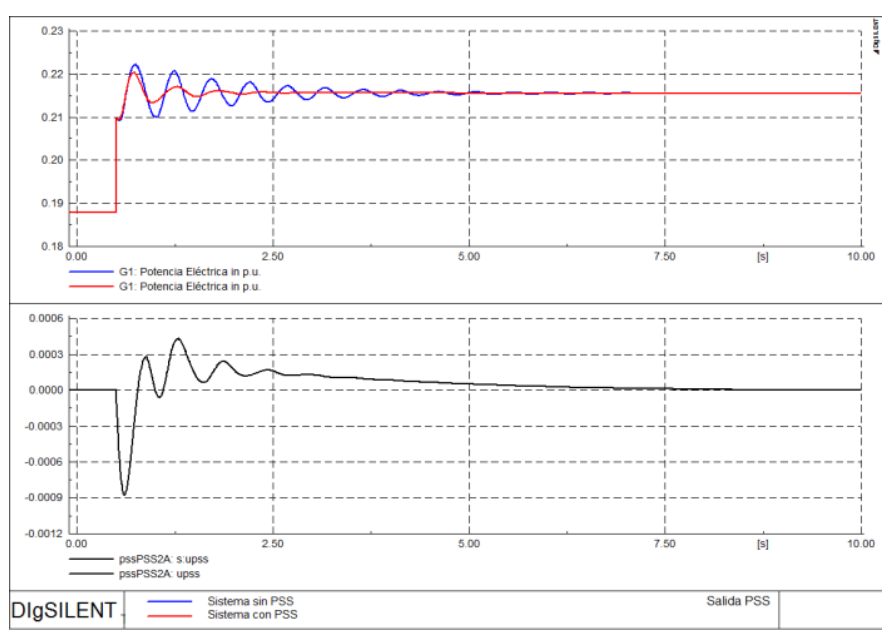

Fig. 13 Potencia de salida del PSS con incremento de carga

En la figura 14 se muestra la señal de salida del PSS conjuntamente con la señal de la potencia eléctrica del generador 2. Con esta figura se busca ilustrar el comportamiento del PSS frente a la presencia de oscilaciones y el efecto final que produce sobre las señales del sistema.

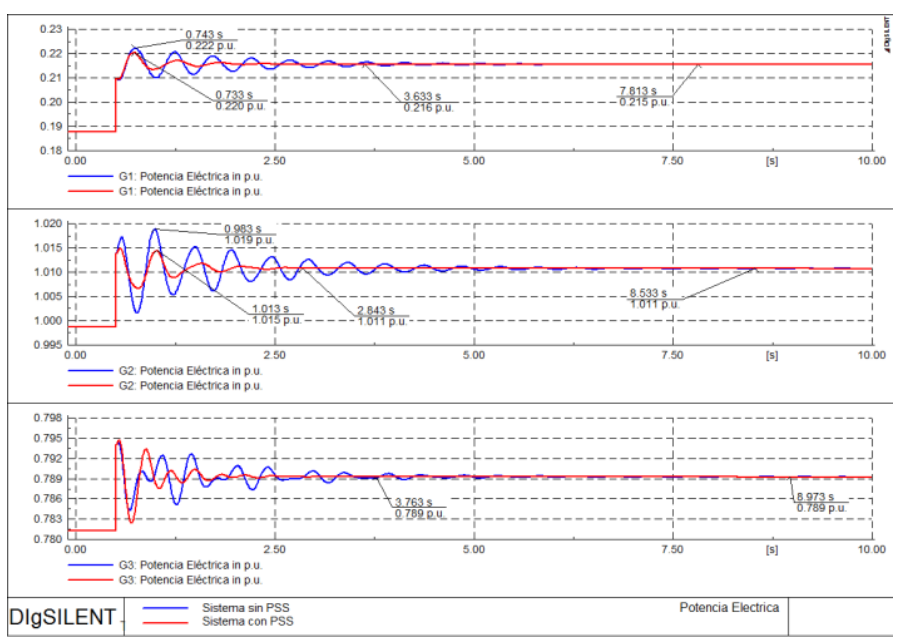

Fig. 14 Potencia eléctrica de los generadores después de falla trifásica

Tabla 3 Índices de desempeño de las señales de la Fig. 14

\begin{tabular}{|c|c|c|c|c|c|c|}
\hline \multicolumn{7}{|c|}{ Potencia activa en los generadores } \\
\hline & \multicolumn{2}{|c|}{ Tiempo de Establecimiento [s] } & \multirow[t]{2}{*}{ Reducción [\%] } & \multicolumn{2}{|c|}{ Sobreimpulso [pu] } & \multirow[t]{2}{*}{ Reducción [\%] } \\
\hline & Sin PSS & Con PSS & & Sin PSS & Con PSS & \\
\hline Generador 1 & 7,813 & 3,633 & 53,501 & 0,007 & 0,005 & 28,571 \\
\hline Generador 2 & 8,533 & 2,843 & 66,682 & 0,008 & 0,004 & 50,000 \\
\hline Generador 3 & 8,973 & 3,763 & 58,063 & $\ldots$ & $\ldots$ & - \\
\hline
\end{tabular}

\section{Falla Trifásica}


Un PSS está diseñado específicamente para corregir los problemas de inestabilidad oscilatoria en un sistema, asociados con pequeñas perturbaciones; sin embargo, este dispositivo también logra amortiguar las oscilaciones ocasionadas por grandes perturbaciones.

Dado que la perturbación de falla trifásica en el sistema es la más grave, se observan oscilaciones de mayor magnitud en las variables del sistema de potencia de las figuras 15 y 16 . Los respectivos índices de desempeño se detallan en las tablas 4 y 5 .

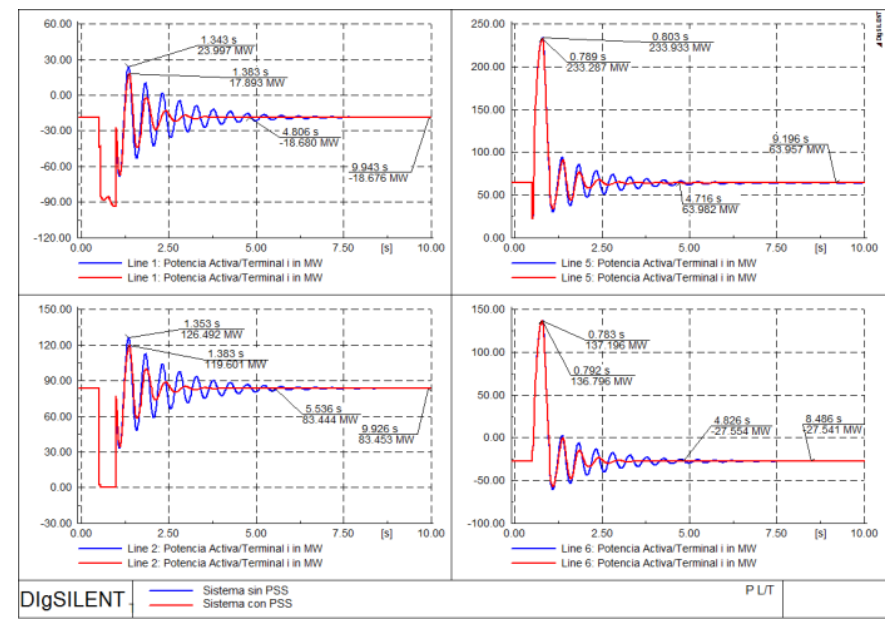

Fig. 15 Potencia eléctrica en las líneas de transmisión después de falla trifásica

Tabla 4 Índices de desempeño de las señales de la Fig. 15

\begin{tabular}{|c|c|c|c|c|c|c|}
\hline \multicolumn{7}{|c|}{ Potencia activa en las líneas de transmisión } \\
\hline & \multicolumn{2}{|c|}{ Tiempo de Establecimiento [s] } & \multirow[t]{2}{*}{ Reducción [\%] } & \multicolumn{2}{|c|}{ Sobreimpulso [MW] } & \multirow{2}{*}{ Reducción $[\%$} \\
\hline & Sin PSS & Con PSS & & Sin PSS & Con PSS & \\
\hline Línea 1 & 9,943 & 4,806 & 51,664 & 42,673 & 36,569 & 14,304 \\
\hline Linea 2 & 9,926 & 5,536 & 44,227 & 43,039 & 36,148 & 16,011 \\
\hline Línea 5 & 9,196 & 4,716 & 48,717 & 169,976 & 169,33 & 0,380 \\
\hline Línea 6 & 8,486 & 4,826 & 43,130 & 164,737 & 164,337 & 0,243 \\
\hline
\end{tabular}

Tabla 5 Índices de desempeño de las señales de la Fig. 16

\begin{tabular}{|c|c|c|c|c|c|c|}
\hline \multicolumn{7}{|c|}{ Potencia activa en los generadores } \\
\hline & \multicolumn{2}{|c|}{ Tiempo de Establecimiento [s] } & \multirow[t]{2}{*}{ Reducción [\%] } & \multicolumn{2}{|c|}{ Sobreimpulso [pu] } & \multirow[t]{2}{*}{ Reducción [\% } \\
\hline & Sin PSS & Con PSS & & Sin PSS & Con PSS & \\
\hline Generador 1 & 9,656 & 4,406 & 54,370 & 0,336 & 0,315 & 6,250 \\
\hline Generador 2 & 9,163 & 4,186 & 54,316 & 0,367 & 0,291 & 20,708 \\
\hline Generador 3 & 8,496 & 5,466 & 35,664 & 0,118 & 0,09 & 23,729 \\
\hline
\end{tabular}

De manera análoga, en la figura 17 se muestra la señal de salida del PSS. Se puede apreciar un pequeño corte en la señal, debido a los limitadores del estabilizador, que evitan excursiones indeseables de voltaje.

\section{CONCLUSIONES}

La inestabilidad de ángulo en sistemas de potencia puede tener su origen en la falta de torque sincronizante o de amortiguamiento. La falta de torque sincronizante se puede corregir mediante la aplicación de AVRs; sin embargo, estos dispositivos son los causantes de la falta de torque de amortiguamiento.

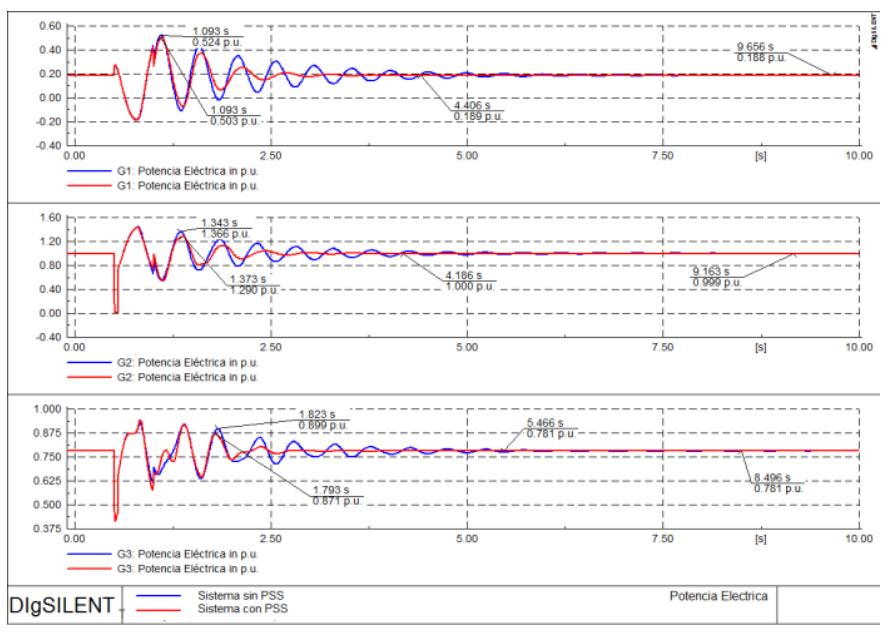

Fig. 16 Potencia eléctrica de los generadores después de falla trifásica

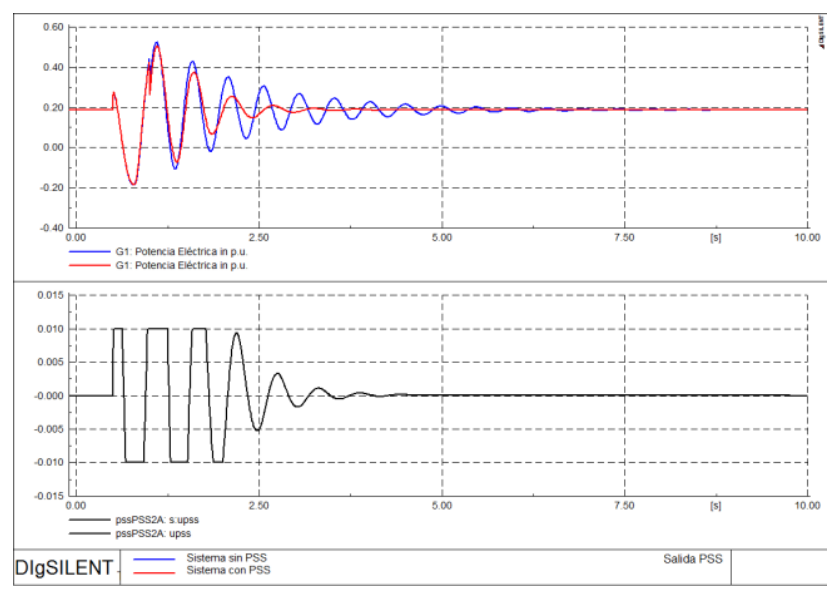

Fig. 17 Potencia de salida del PSS después de falla trifásica

Los valores propios de un sistema permiten conocer los modos de oscilación y su grado de amortiguamiento.

La ubicación más adecuada de un PSS se determina mediante el análisis conjunto de los factores de participación de los generadores del sistema y la respuesta de frecuencia en el modelo Generador - Barra Infinita.

Con el modelo Generador - Barra Infinita resulta evidente que sólo se realiza la sintonización del PSS para un modo de oscilación local. Esto se debe a que de por sí, el modelo considera solamente un generador y una barra infinita, es decir, un generador oscilando contra el resto del sistema.

Los modos de oscilación en un sistema de potencia pueden constituir un problema al tener un amortiguamiento pobre, lo que se traduce en un tiempo de decaimiento muy grande o también en una amplitud de gran magnitud. 
Tomando como referencia los índices de desempeño de pequeña señal se puede observar en las simulaciones en el dominio del tiempo, que la sintonización del PSS tiene el efecto deseado dentro del sistema, al lograr reducir en todos los casos en más del 30\% el tiempo de establecimiento de las señales y una reducción considerable del sobreimpulso.

\section{REFERENCIAS}

[1] KUNDUR Prabha, Power System Stability and Control, McGraw - Hil, 1994.

[2] MURDOCH A., VENKATARAMAN S., LAWSON R.A., PEARSON W.R., Integral of Accelerating Power Type PSS, Part 1 \& 2, IEEE Transactions on Energy Conversion, 1999.

[3] BIKASH Pal, BALARKO Chaudhuri, Robust Control in Power Systems, Springer, 2005.

[4] KUNDUR P., KLEIN M., ROGERS G.J., Application of Power System Stabilizers for Enhancement of Overall Systems, IEEE Transactions, 1999.

[5] TANG Bixiang, Parameter Tuning and Experimental Results of Power System Stabilizer, Tesis de Maestría, 2011.

[6] IEEE COMMITTEE REPORT, Excitation System Models for Power System Stability Studies, IEEE Transactions on Power Apparatus and Systems, 1981.
[7] BÉRUBÉ G.R., HAJAGOS L.M., Accelerating - Power Based Power System Stabilizers, 2007.

[8] PADIYAR K.R., Power System Dynamics Stability and Control, BS Publications, 2008.

[9] ANDERSON P.M., FOUAD A.A., Power System Control and Stability, IEEE PRESS, 2003.

\section{BIOGRAFÍAS}

Pablo Verdugo Rivadeneira, nació en la ciudad de Quito en 1987. Realizó sus estudios secundarios en el Colegio Nacional Experimental Juan Pío Montufar y sus estudios superiores en la Escuela Politécnica Nacional. Actualmente trabaja en el CENACE.

Jesús Játiva Ibarra, realizó sus estudios superiores en la EPN, donde se graduó de Ingeniero Eléctrico, en 1981. Realizó estudios de postgrado en la Universidad de Texas en Arlington de USA, donde obtuvo el grado de Master of Science in Electrical Engineering en 1988, y posteriormente el título de Doctor of Philosophy en 1991. Cuenta con más de treinta años de experiencia en actividades de Planificación, Operación, Administración y Economía de sistemas eléctricos de potencia. Actualmente es Profesor Principal de la EPN. 